\title{
Thermal Behavior of Langmuir-Blodgett Film of Poly(tert-butyl methacrylate) by Principal Component Analysis Based Two-Dimensional Correlation Spectroscopy
}

\author{
Young Mee Jung* and Seung Bin Kim ${ }^{\dagger}$ \\ Department of Chemistry, Kangwon National University, Chunchon 200-701, Korea. *E-mail: ymjung@kangwon.ac.kr \\ † Department of Chemistry, Pohang University of Science and Technology, Pohang 790-784, Korea \\ Received November 9, 2005
}

\begin{abstract}
This paper demonstrates details of thermal behavior of Langmuir-Blodgett (LB) film of poly(tert-butyl methacrylate) (PtBMA) by using the principal component analysis based two-dimensional correlation spectroscopy (PCA2D) through eigenvalue manipulating transformation (EMT). By uniformly lowering the power of a set of eigenvalues associated with the original data, the smaller eigenvalues becomes more prominent and the subtle contribution from minor components is now highlighted much more strongly than the original data. Thus, the subtle difference of thermal behavior of LB film of PtBMA from minor components, which is not readily detectable in the conventional $2 \mathrm{D}$ correlation analysis, is much more noticeable than the original data. PCA2D correlation spectra with EMT operation for the temperature-dependent IR spectra of LB film of PtBMA reveal the hidden property of phase transition processes during heating.
\end{abstract}

Key Words : Two-dimensional (2D) correlation spectroscopy, PCA2D correlation spectroscopy, Eigenvalue manipulation transformation (EMT), Poly(tert-butyl methacrylate) (PtBMA), Phase transition

\section{Introduction}

We have proposed promising approaches to generalized two-dimensional (2D) correlation spectroscopy, which demonstrate a completely new way of directly integrating the relatively well-established chemometrics techniques like principal component analysis (PCA) into 2D correlation spectroscopy. ${ }^{1-6}$ Both 2D correlation spectroscopy ${ }^{7-9}$ and PCA $^{10-12}$ have been utilized extensively, but often independently, in spectral analyses in many fields of spectroscopic studies. In our previous papers, ${ }^{1-6}$ principal component analysis-based 2D (PCA2D) correlation spectroscopy, which provides fully integrated true combination of these two powerful spectral data analysis techniques, has been proposed as a method to improve the data quality for $2 \mathrm{D}$ correlation spectroscopy. In PCA2D correlation spectroscopy, PCA treatment of spectral data set becomes an essential and integral part of the subsequent 2D correlation spectroscopy. We have established that, by simply suppressing the influence of minor principal components (PCs), noise contribution to the reconstructed data matrix and consequently to the $2 \mathrm{D}$ correlation spectra calculated from such a data matrix, is effectively reduced. Additional modification of $2 \mathrm{D}$ correlation spectra to enhance certain correlational features, which is possible by systematically manipulating the eigenvalues, was also demonstrated.

We have formulated the reconstructed data matrix $\mathbf{A}^{*}$, which no longer contains the noise contributions, from a few selected significant scores and loadings derived from PCA of the original set of perturbation-dependent spectra $\mathbf{A}$.

$$
\mathbf{A}^{*}=\mathbf{W} \mathbf{V}^{\mathbf{T}} \text {. }
$$

where, $\mathbf{W}$ and $\mathbf{V}$ are a score matrix and a loading matrix, respectively. The notation $\mathbf{V}^{\mathbf{T}}$ stands for the transpose of $\mathbf{V}$.
This reconstructed data matrix $\mathbf{A}^{*}$ is utilized for the calculation of 2D correlation spectra instead of the original data matrix. The 2D correlation spectroscopy of the PCAreconstructed data matrix accentuated the most important features of synchronicity and asynchronicity without being hampered by noise.

The PCA-reconstructed data matrix $\mathbf{A}^{*}$ is also expressed by the singular value decomposition (SVD), ${ }^{10-12}$

$$
\mathbf{A}^{*}=\mathbf{U S} \mathbf{V}^{\mathbf{T}} \text {. }
$$

and

$$
\mathbf{S}=\mathbf{L}^{1 / 2}
$$

where $\mathbf{U}, \mathbf{S}$, and $\mathbf{V}$ are a orthonormal matrix, a diagonal matrix, and a loading matrix, respectively. Here $\mathbf{L}=\mathbf{W}^{\mathbf{T}} \mathbf{W}$ is a diagonal matrix where each diagonal element corresponds to the eigenvalue of principal component. The score matrix $\mathbf{W}$ is expressed in the form $\mathbf{W}=\mathbf{U} \mathbf{S}$ and can be obtained directly from $\mathbf{W}=\mathbf{A} \mathbf{V}$.

The new transformed data matrix $\mathbf{A}^{* *}$ will be obtained by manipulating and replacing eigenvalues of $\mathbf{A}^{*}$ as

$$
\mathbf{A}^{* *}=\mathbf{U} \mathbf{S}^{* *} \mathbf{V}^{\mathbf{T}}
$$

where $\mathrm{S}^{* *}$ is given by varying the corresponding eigenvalues in $\mathrm{S}$ by raising or lowering them to the power of $m$.

$$
\mathbf{S}^{* *}=\mathbf{S}^{m}
$$

This new EMT-reconstructed data matrix $\mathbf{A}^{* *}$ will be used instead of $\mathbf{A}^{*}$ for the calculation of the enhanced 2D correlation spectra.

By uniformly raising the power of a set of original eigenvalues, the influence of factors associated with major eigenvalues becomes more prominent, while the minor eigenvectors primarily arising from the noise component are 
no longer strongly represented. Thus, this transformation of data matrix becomes a gradual noise reduction scheme with attractive flexibility of continuously fine-tuning the balance between the desired noise suppression and retention of pertinent spectral information. However, by uniformly lowering the power of a set of eigenvalues associated with the original data, the smaller eigenvalues becomes more prominent and the contributions of minor components become amplified. Thus, much more subtle difference of spectral behavior for each component is now highlighted.

We have previously reported the overall reorientation of functional groups which is sensitive to conformational changes of the LB film of PtBMA with temperature increasing by using conventional 2D correlation spectroscopy and principal component analysis. ${ }^{13-15}$ The observation that the strongest intensity change below the glass transition temperature occurs at $1137 \mathrm{~cm}^{-1}$, therefore, indicates that the reorganization of this band is potentially the mechanism underlying the $\beta$-transition. While, for above the glass transition temperature, the intensity of bands at 1162 and $1148 \mathrm{~cm}^{-1}$ change greatly, suggesting the C-O group in the LB film undergoes mainly local reorientation.

In present study, we employed PCA2D correlation spectroscopy through EMT technique, to better understand details of thermal behavior of LB film of PtBMA during heating. By lowering the power of a set of eigenvalues associated with the original data, the subtle difference in the thermal responses of LB film of PtBMA, which are difficult to observe by conventional $2 \mathrm{D}$ correlation analysis, are enhanced much more strongly than the original data.

\section{Experimental Section}

The temperature-dependent external reflection absorption FTIR spectra of an LB film of PtBMA were analyzed in the present study. The detail experimental procedure was described elsewhere. ${ }^{13-15}$

Prior to PCA calculation, the mean centering operation was applied to the data matrix. To preserve the amplitude

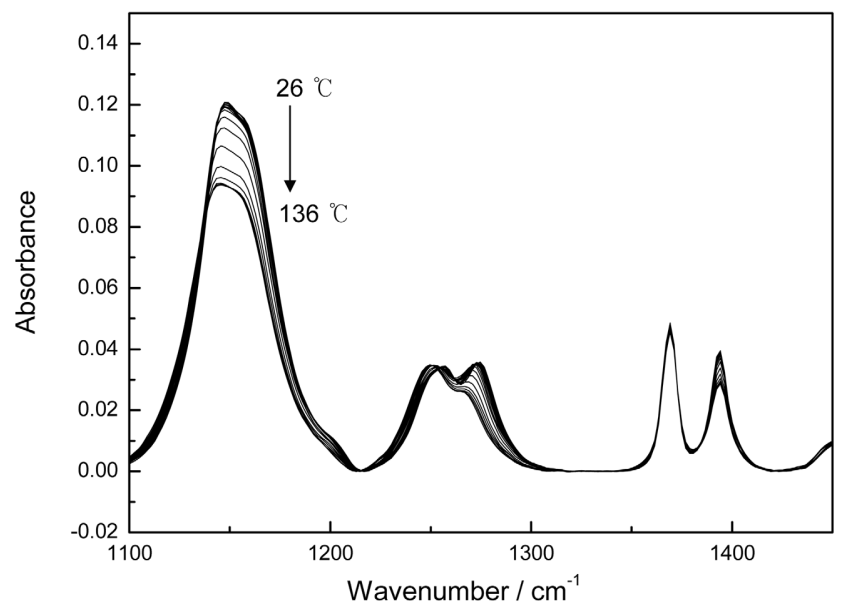

Figure 1. FTIR spectra of a 50-layer LB film of PtBMA at various temperatures $\left(26-136^{\circ} \mathrm{C}\right)$. information of the variation of spectral intensities, which becomes important later in 2D correlation analysis, other steps commonly used in PCA such as normalization scaling of data according to the standard deviation, were not carried out. PCA analysis was performed in the Pirouette software (Infometrix Inc.).

Synchronous and asynchronous PCA2D correlation spectra were obtained using the same software as those described previously. ${ }^{1}$ To minimize the contribution of noise
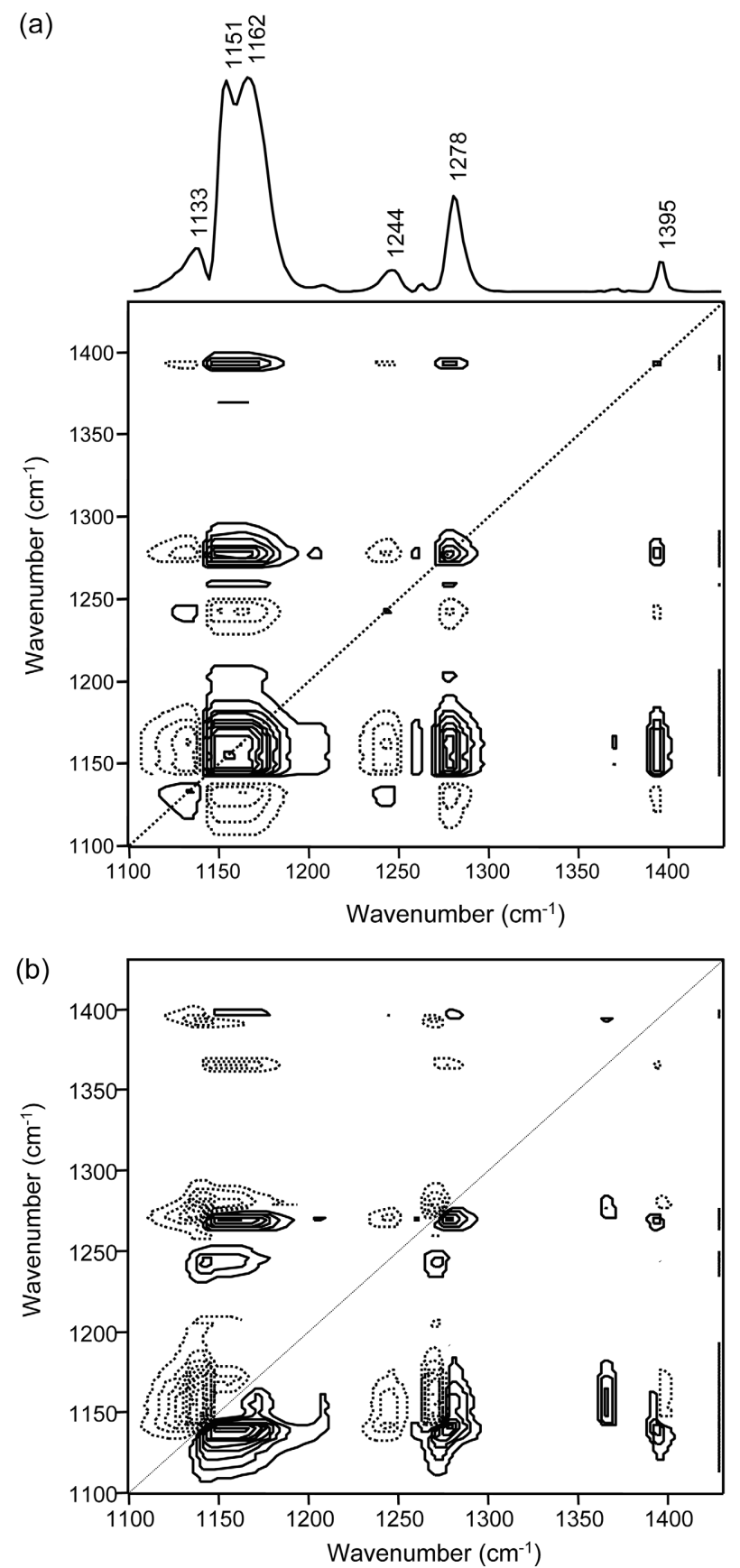

Figure 2. Conventional synchronous (a) and asynchronous (b) 2D correlation spectra obtained from temperature-dependent spectral changes of an LB film of PtBMA in the $1100-1430 \mathrm{~cm}^{-1}$ region. Solid and dashed lines represent positive and negative cross peaks, respectively. 

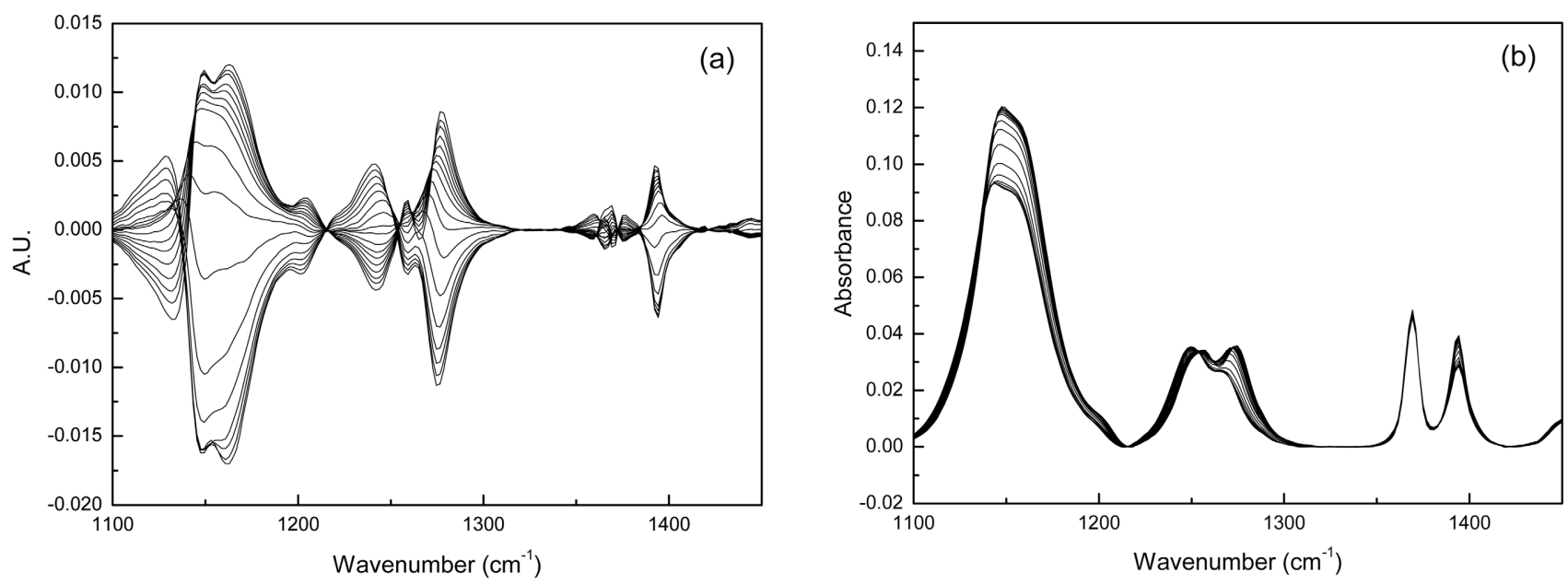

Figure 3. Spectra of PCA-reconstructed data from loadings and scores of PC1, PC2 and PC3 (a) and the PCA-reconstructed spectra with average spectrum added back (b).

component in the EMT-reconstructed data matrix, only the dominant three principal components are used, and the rest are truncated, in this study. We systematically vary the value of $m$, as $m=1,1 / 2,1 / 3$, and 0 , for $2 \mathrm{D}$ correlation spectra
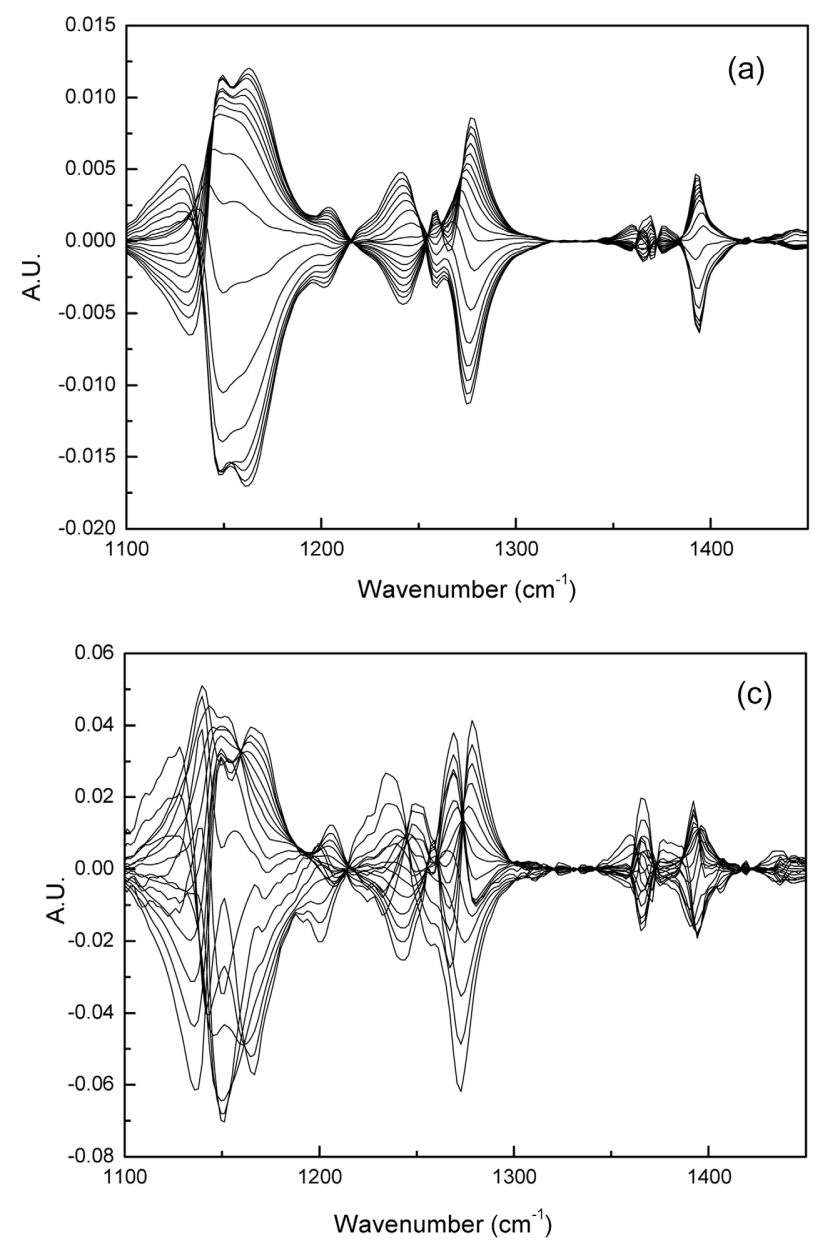

generated from this new EMT-reconstructed data matrix. All the reconstructed data matrixes used in this study were calculated using MATLAB software (Version 6, The Math Works Inc.).
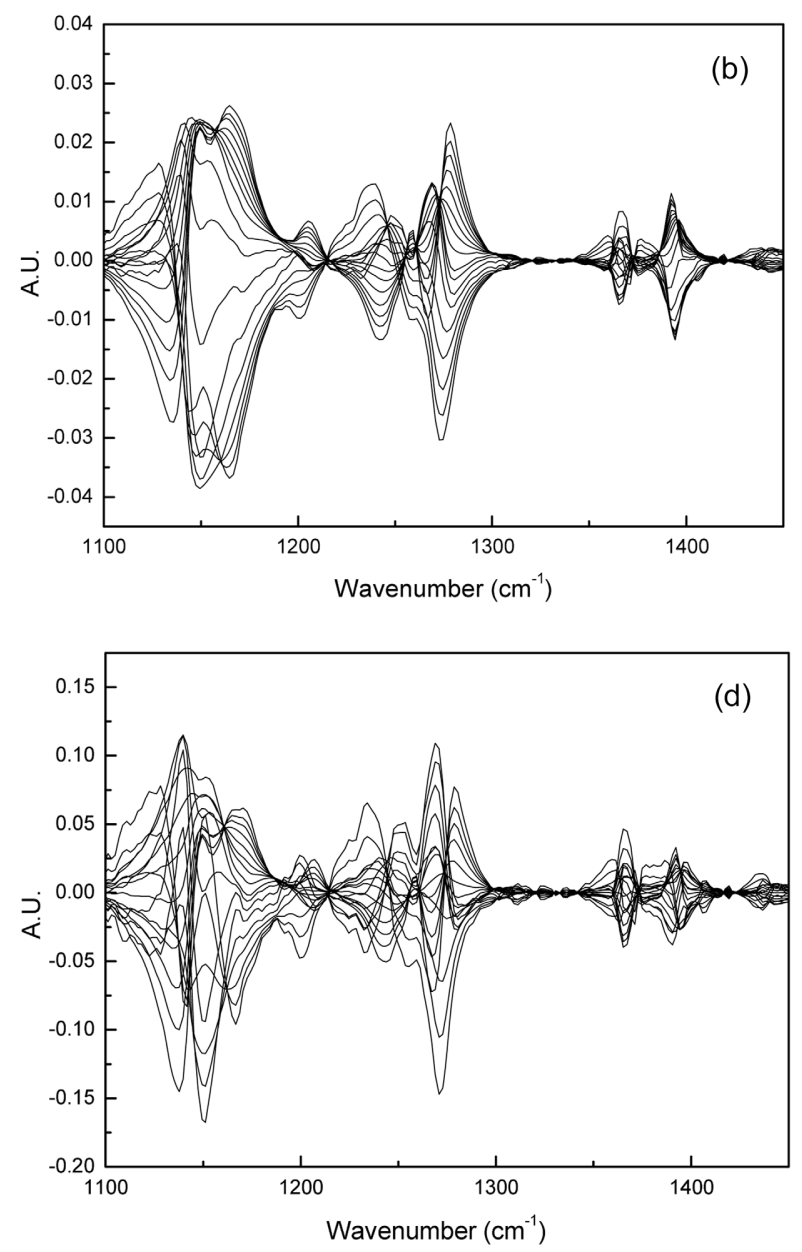

Figure 4. Spectra of the EMT-reconstructed data obtained by replacing eigenvalues from loadings and scores of PC1, PC2 and PC3. Each spectrum is obtained by varying the value of power parameter $m$, as $m=1$ (a), 1/2 (b), 1/3 (c), and 0 (d). 
(a)

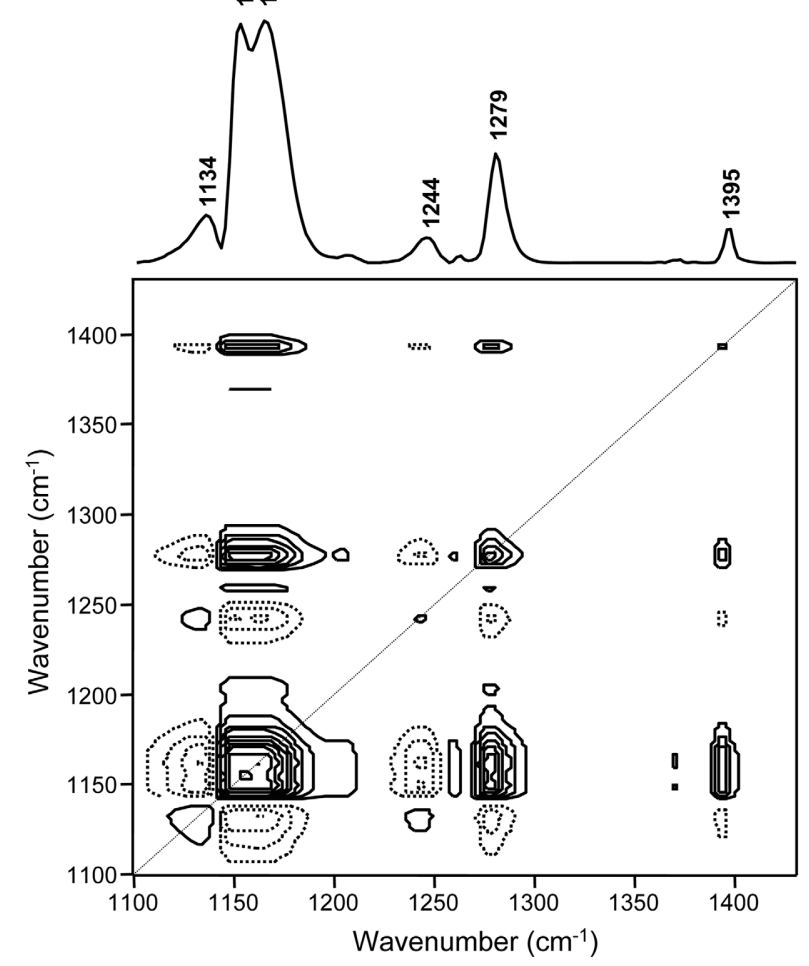

(c)

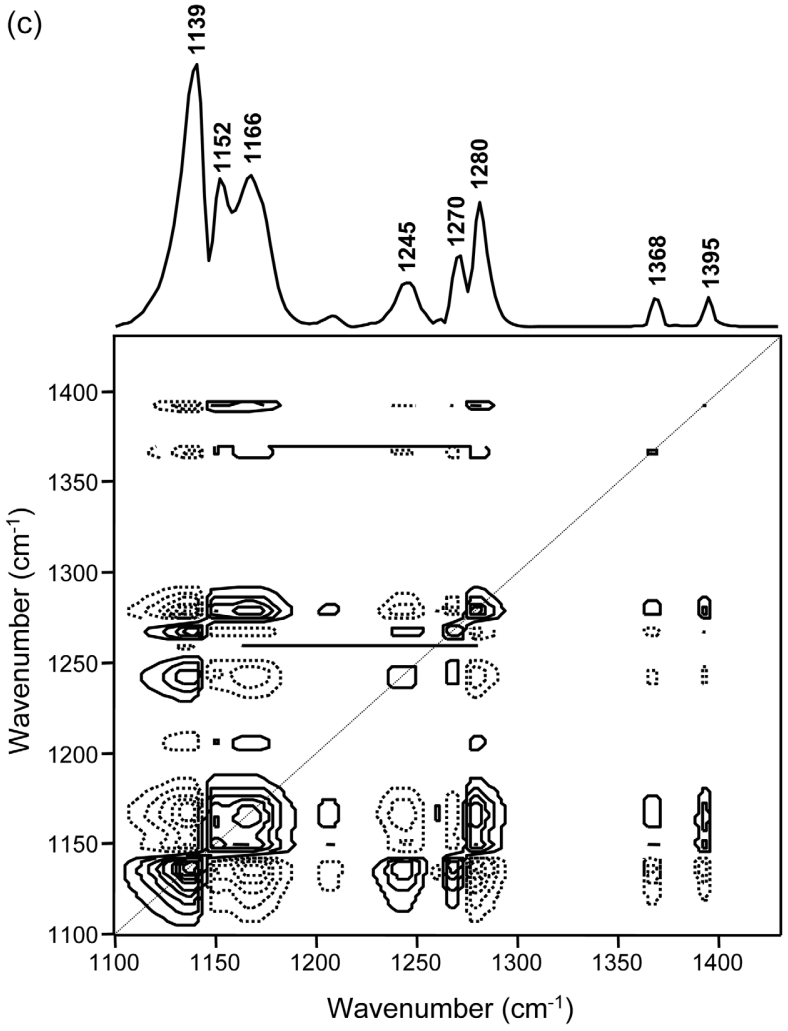

(b)

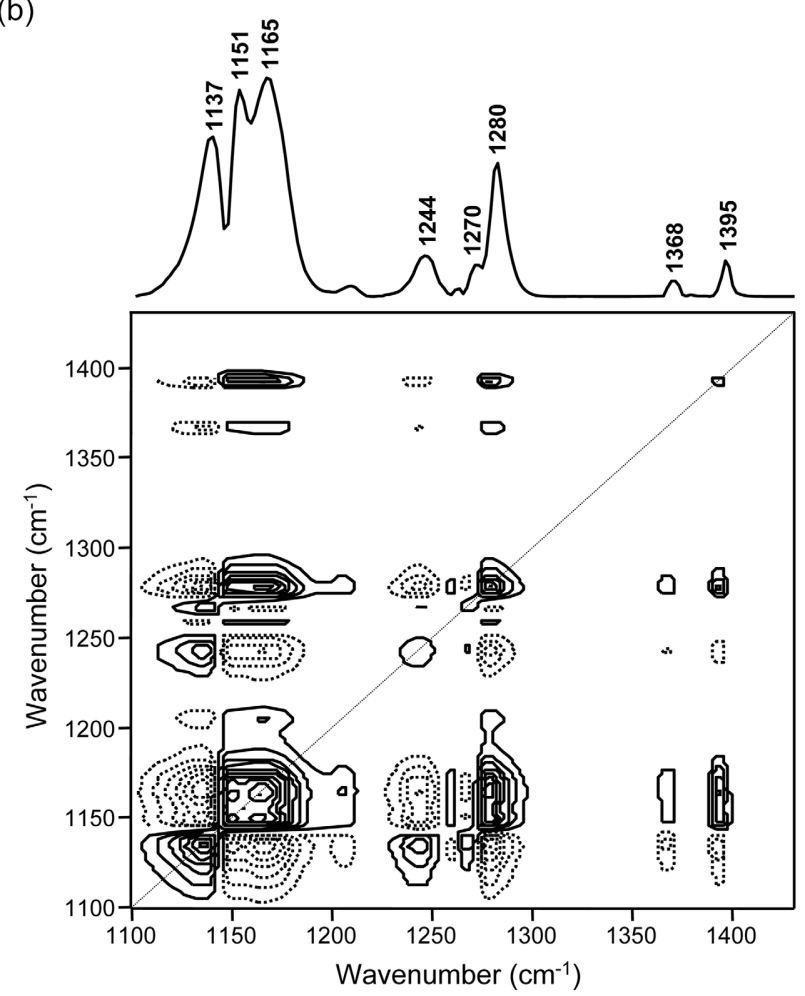

(d)

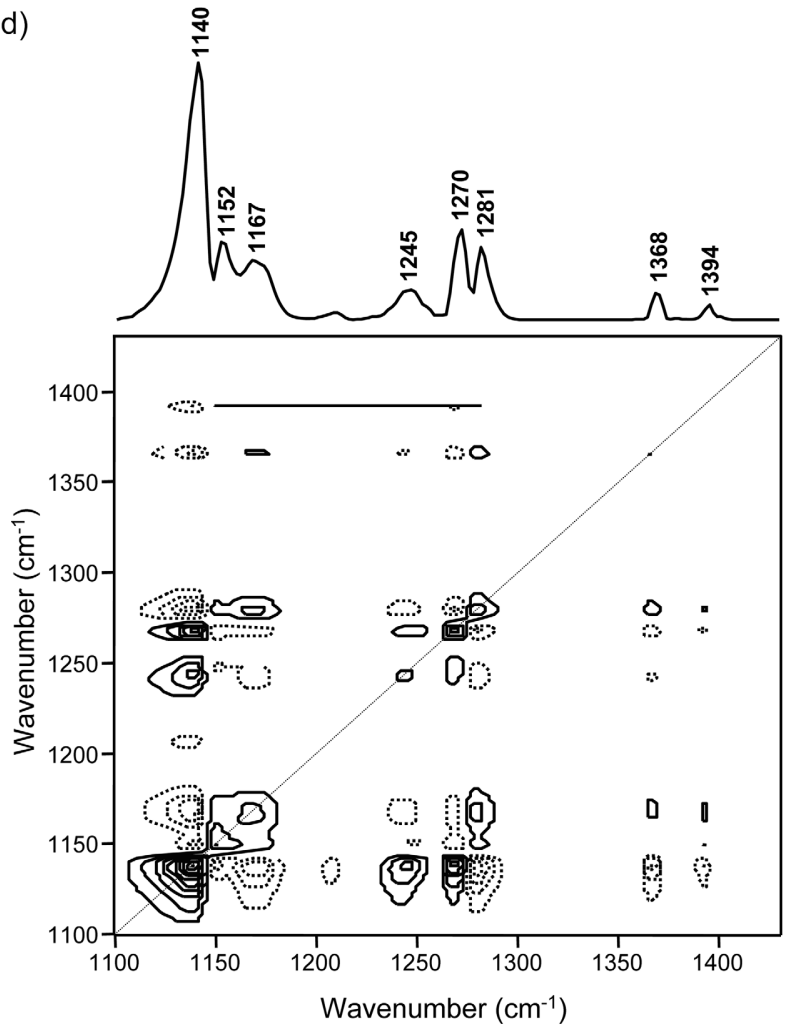

Figure 5. Synchronous 2D correlation spectra constructed from the EMT-reconstructed data obtained by varying the value of power parameter $m$, as $m=1$ (a), 1/2 (b), 1/3 (c), and 0 (d). Solid and dashed lines represent positive and negative cross peaks, respectively.

\section{Results and Discussion}

Figure 1 shows the original spectra used in the present study, which is external reflection absorption FTIR spectra of an LB film of PtBMA measured over a temperature range of 26 to $136{ }^{\circ} \mathrm{C}$. The assignment of the observed bands and 
the detail information of these spectra were given in the preceding papers. ${ }^{13-15}$ Conventional synchronous and asynchronous 2D correlation spectra constructed from the temperature-dependent IR spectra in Figure 1 are displayed in Figure 2(a) and (b), respectively. The analysis of synchronous and asynchronous 2D correlation spectra was described in more detail previously, ${ }^{13-15}$ so no further interpretation will be given here.

The original spectral data set in Figure 1 was decomposed into the scores and loadings by a standard PCA analysis. The obtained scores of PCA factor 1 (PC1), factor 2 (PC2), and factor 3 (PC3) accounts for $97.09 \%, 2.29 \%$, and $0.32 \%$, respectively, of the total variance. The scores and loadings plots of PCs and the detail interpretation of PCA analysis of the temperature-dependent IR spectral changes were also described in the preceding paper. ${ }^{15}$ In this contribution, the reconstructed data matrix $\mathbf{A}^{*}$ obtained by eq. (1) was used instead of the original raw spectral data matrix $\mathbf{A}$ for the $2 \mathrm{D}$ correlation analysis. Figure 3(a) depicts spectra of recon- structed data represented in the matrix $\mathbf{A}^{*}$ from loadings and scores of PC1, PC2, and PC3. The PCA-reconstructed spectra with average spectrum added back, $\mathbf{A}^{*}+\overline{\mathbf{A}}$, were shown in Figure 3(b) for easier comparison with Figure 1, where $\overline{\mathbf{A}}$ is the reference spectrum obtained by averaging the raw spectral data over the entire temperature. It is noted that the spectra of $\mathbf{A}^{*}+\overline{\mathbf{A}}$ look virtually indistinguishable from the first set of raw spectra in Figure 1, suggesting that most of the pertinent information without noise component is retained in the PCA-reconstructed data.

The new EMT-reconstructed data matrix $\mathbf{A}^{* *}$ obtained by eqs. (4) and (5) was used instead of the PCA-reconstructed data matrix $\mathbf{A}^{*}$ for the $2 \mathrm{D}$ correlation analysis. Figure 4 shows a series of EMT-reconstructed spectral data $\mathbf{A}^{* *}$ obtained by replacing eigenvalues of $\mathbf{A}^{*}$. Clearly from spectra in Figure 4, changing the power of eigenvalues even at $m=1 / 2$ to $m=1 / 3$ has very profound effects on the reconstructed spectra. As noted before, by lowering the power parameter $m$, the contribution of the first loading to
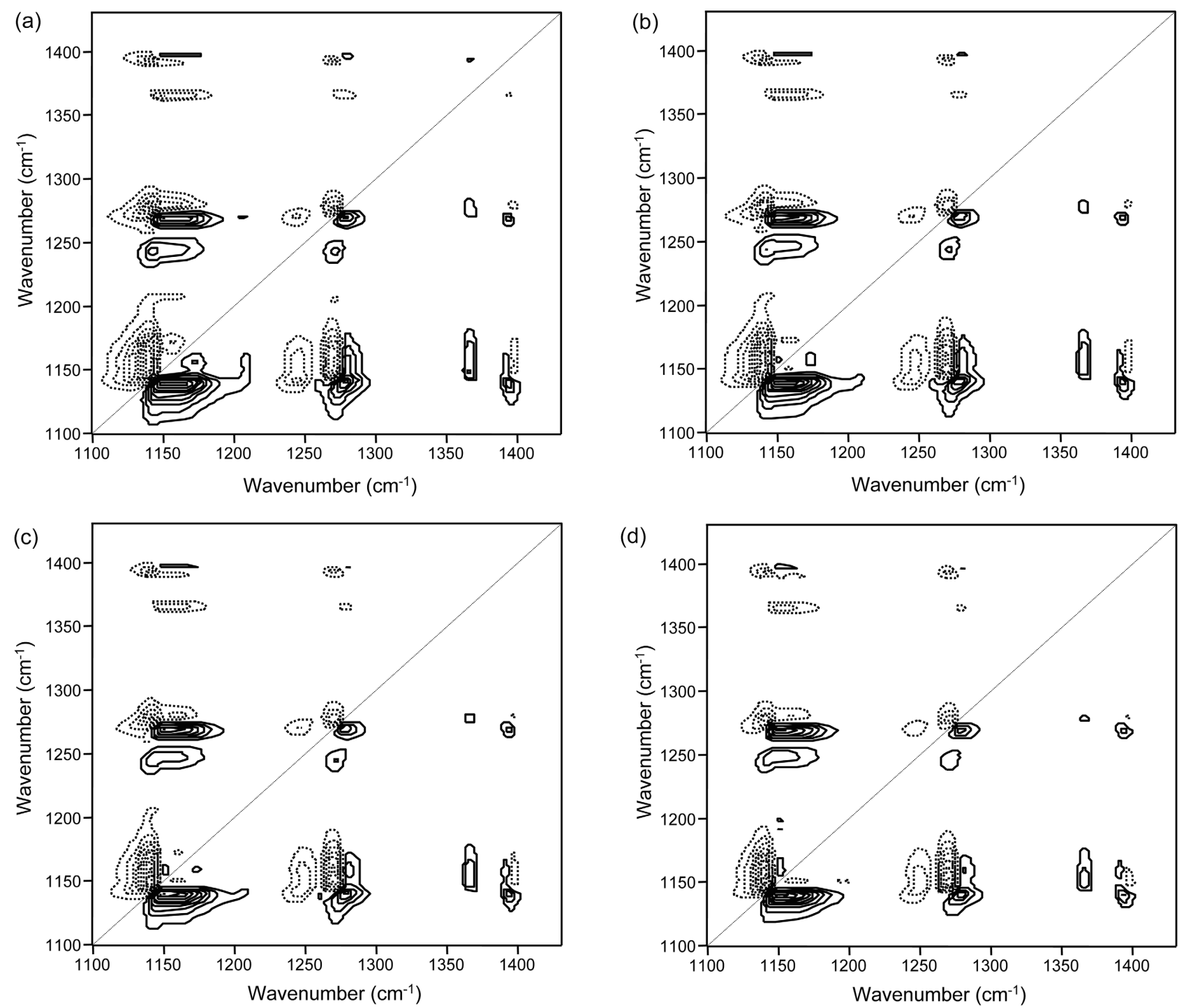

Figure 6. Asynchronous $2 \mathrm{D}$ correlation spectra constructed from the EMT-reconstructed data obtained by varying the value of power parameter $m$, as $m=1$ (a), 1/2 (b), 1/3 (c), and 0 (d). Solid and dashed lines represent positive and negative cross peaks, respectively. 
the reconstructed data matrix become much less and that of the minor but still significant components, which often contain the most interesting information about spectral data set, is enhanced greatly. Thus, the similarity and difference in dynamic variation of spectra intensities will be greatly accentuated by magnifying the minor eigenvalues.

If the parameter $m$ is 1 , the EMT-reconstructed data matrix is the same as noise truncated PCA-reconstructed data matrix, i.e., $\mathbf{A}^{* *}=\mathbf{A}^{*}$ (Figure 4(a)).

Figure 5 displays synchronous $2 \mathrm{D}$ correlation spectra from the EMT-reconstructed data matrix $\mathbf{A}^{* *}$ obtained by replacing the eigenvalues of $\mathbf{A}^{*}$. Power spectra extracted along the diagonal line of the synchronous spectra are also shown on the top of each synchronous 2D correlation spectra. Figure 6 depicts the corresponding asynchronous $2 \mathrm{D}$ correlation spectra. Particularly striking is that the effect of eigenvalue manipulation by accentuation of minor factor is much more noticeable on the synchronous $2 \mathrm{D}$ correlation spectrum. Our EMT operation tends to exaggerate the effect of minor PCs and reduce that of major PCs. Thus, the subtle difference in thermal behavior of LB film of PtBMA, which is difficult to observe by ordinary $2 \mathrm{D}$ analysis, can be greatly emphasized.

It is noted that the highly EMT-enhanced synchronous 2D correlation spectrum (i.e., at the power parameter $m=0$, Figure 4(d)) shows the prominence of bands at 1368 and $1270 \mathrm{~cm}^{-1}$ barely observable in original synchronous $2 \mathrm{D}$ correlation spectrum (Figure 2(a)). It provides the useful information about the transition processes occurring in this LB film of PtBMA. As we have reported previously, ${ }^{13}$ we can determine only a rough estimation of the glass transition temperature from scores of PC2. The plot of the PC2 loading vector shows that the transition occurring at the glass transition temperature is mainly correlated with absorptions at 1368,1270 , and $1139 \mathrm{~cm}^{-1}$ assigned to $\mathrm{CH}_{3}$ bending vibration of tert-butyl group, the stretching mode of C-C-O, and the bending mode of the ${ }^{\mathrm{bb}} \mathrm{C}-\mathrm{C}-\mathrm{O}$ and ${ }^{\mathrm{bb}} \mathrm{C}-\mathrm{C}=\mathrm{O}$ bonds connected to the backbone (bb) chain, respectively. The new correlation peaks observed in the EMT-enhanced synchronous 2D spectrum clearly reflect the coordinated intensity changes of these bands, as the temperature of the system is increased. Thus it reveals the hidden property of glass transition temperature.

\section{Conclusion}

In present study, we have successfully demonstrated that the thermal behavior of LB film of PtBMA is more clearly understood by PCA2D correlation spectroscopy through EMT operation. By lowering the power of a set of eigenvalues associated with the original data, the contribution of the minor but potentially more interesting factors is accentuated much more greatly than original data. Thus subtle difference in the variational behavior of spectral intensities is much more noticeable than original data.

Based on the PCA2D correlation spectra for the EMTreconstructed spectral data matrix $\mathbf{A}^{* *}$, obtained by accentuating the minor eigenvalues, it is found that the subtle differences in the thermal responses, which are difficult to observe by conventional 2D correlation analysis, are accentuated much more strongly than the original data. We then shed light on understanding hidden thermal behavior of LB film of PtBMA. Thus the PCA2D correlation spectroscopy through EMT operation would certainly open way for studying phase behavior of polymers.

Acknowledgment. This study was supported by the Research Grant from Kangwon National University.

\section{References}

1. Jung, Y. M.; Shin, S. H.; Kim, S. B.; Noda, I. Appl. Spectrosc. 2002, 56, 1562 .

2. Jung, Y. M. Vib. Spectrosc. 2002, 36, 279.

3. Jung, Y. M.; Kim, S. B.; Noda, I. Appl. Spectrosc. 2003, 57, 557.

4. Jung, Y. M.; Kim, S. B.; Noda, I. Appl. Spectrosc. 2003, 57, 564.

5. Jung, Y. M.; Kim, S. B.; Noda, I. Appl. Spectrosc. 2003, 57, 850.

6. Jung, Y. M. Bull. Korean Chem. Soc. 2003, 24, 1345.

7. Noda, I. Appl. Spectrosc. 1993, 47, 1329.

8. Noda, I.; Dowrey, A. E.; Marcott, C.; Story, G. M.; Ozaki, Y. Appl. Spectrosc. 2000, 54, 236A.

9. Noda, I.; Ozaki, Y. Two-Dimensional Correlation Spectroscopy: Applications in Vibrational Spectroscopy; John Wiley \& Sons, Inc.: New York, 2004.

10. Malinowski, E. R. Factor Analysis in Chemistry, 2nd ed; Wiley: New York, 1991.

11. Martens, H.; Næs, T. Multivariate Calibration; John Wiley \& Sons: New York, 1991.

12. Vandeginste, B. G. M.; Massart, D. L.; Buydens, L. M. C.; De Jong, S.; Lewi, P. J.; Smeyers-Verbeke, J. Handbook of Chemometrics and Qualimetrics: Part B; Elsevier Science B. V.: Amsterdam, The Netherlands, 1998.

13. Jung, Y. M.; Shin, S. H.; Czarnik-Matusewicz, B.; Noda, I.; Kim, S. B. Appl. Spectrosc. 2002, 56, 1586.

14. Shin, S. H.; Jung, Y. M.; Lee, J.; Chang, T.; Ozaki, Y.; Kim, S. B. Langmuir 2002, 18, 5523.

15. Shin, S. H.; Jung, Y. M.; Chang, T.; Ozaki, Y.; Kim, S. B. Vib. Spectrosc. 2002, 29, 79. 